\title{
Annexin A2 inhibits the migration of PASMCs stimulated with HPS rat serum by down-regulating the expression of paxillin
}

\author{
Yang Chen ${ }^{1}$, Xinrong Wen ${ }^{1}$, Zhi Wang, Jing Zeng, Bin Chen, Yong Yang, Kaizhi Lu, Jianteng \\ $\mathrm{Gu}^{* *}, \mathrm{Bin} \mathrm{Yi}^{*}$ \\ Department of Anesthesia, Southwest Hospital, the Third Military Medical University, Chongqing \\ 400038, P.R. China \\ Corresponding auther at: Department of Anesthesia, Southwest Hospital, the Third Military \\ Medical University, Chongqing 400038, P.R. China. Fax: +86 2368765371. \\ *E-mail address: yibin1974@163.com (B. Yi). \\ **E-mail address: gjt1976@163.com(J.Gu ). \\ ${ }^{1}$ Yang Chen and Xinrong Wen contributed equally to this study
}

\begin{abstract}
Hepatopulmonary syndrome (HPS) has been classically associated with intrapulmonary vasodilatation (IPVD) and pulmonary vascular remodelling (PVR), which are the key pathophysiological components of HPS and concerned frequently in the studies of HPS. Little is known about the relevance of pulmonary artery smooth muscle cells (PASMCs) migration or the molecular mechanisms of PVR in HPS. Annexin A2 (ANXA2) plays crucial role in HPS-associated PVR and might activate the activity of paxillin which as a regulatory protein participates in the regulation of PASMCs function in PVR. In addition, it has been identified that ANXA2 could influence the cells migration by some important signaling pathways in many diseases, including lung cancer, pulmonary hypertensionand and liver cancer. In this study, we performed scratch wound motility assay, modified boyden chamber, reverse transcription PCR, western blot and co-immunoprecipitation to determine the role of ANXA2 on HPS-associated PVR. We found that HPS rat serum from a common bile duct ligation (CBDL) rat model enhanced the migration of PASMCs and increased the expression of ANXA2 in PASMCs. We reported that ANXA2 and paxillin could form a co-immunoprecipitation. After silencing ANXA2 with siRNA, we found that the up-regulation of paxillin expression, induced by the HPS rat serum, was reversed. Additionally, we found that down-regulation of ANXA2 could significantly inhibit the migration of PASMCs. These findings indicated that down-regulation of ANXA2 by siRNA results in the inhibition of the aberrant dysregulation of paxillin and migration of PASMCs, which suggesting a potential therapeutic effect on HPS-associated PVR.
\end{abstract}


Keywords: Hepatopulmonary syndrome; Pulmonary artery smooth muscle cells; ANXA2; Paxillin; Migration

\section{Introduction}

Hepatopulmonary syndrome (HPS) is a chronic hepatic complication characterised by a defect in arterial oxygenation induced by intrapulmonary vasodilatation (IPVD) in the setting of chronic liver disease[1,2]. Multiple circulating cytokines and growth factors are released by a diseased liver and contribute to the occurrence of IPVD, which leads to development of hypoxemia. Hypoxic conditions contribute to numerous pulmonary artery smooth muscle cells (PASMCs) migrating from membrane to endometrial of pulmonary vascular, where they proliferate abnormally. These are the main pathophysiological changes of pulmonary vascular remodelling (PVR) [3].

Previous studies on HPS-associated PVR mainly focused on PASMC proliferation. Some proteins, including Annexin A2 (ANXA2) and paxillin, play key regulatory roles in PASMC proliferation $[4,5,6]$. However, so far, PASMC migration has not been a major focus in HPS-associated PVR research. A number of studies have shown that chronic exposure to hypoxia leads to the migration of PASMCs [3,5,7]. Therefore, the pathophysiological mechanisms of increased PASMC migration in HPS-associated PVR require an in-depth study. In addition, previous studies have demonstrated that PASMC migration occurs before the proliferation of these cells [8]. We hypothesised that regulating the migration of PASMCs may be a key upstream target for PVR treatment and provide a new approach for HPS prevention.

ANXA2 is found in many tissues and participates in the regulation of numerous cell functions. It is a key regulator of cell migration and invasion in several cell types. Previous studies have demonstrated that the expression of ANXA2 was increased significantly in PASMCs stimulated with HPS rat serum. Furthermore, it was reported that ANXA2 influenced the proliferation of PASMCs treated with HPS rat serum through the ERK and NF- $\kappa \mathrm{B}$ pathways. Most of the time, proliferation occurred after migration $[9,10,11]$. It was noted that ANXA2 may play a key regulatory role in the increased PASMC migration observed in HPS-associated PVR.

It has been reported that paxillin is a key regulator of adhesion, migration and apoptosis of PASMCs, which it regulates via the AKT and ERK1/2 kinases [6]. A number of studies have 
reported that Annexin A1 (ANXA1) regulates integrin $\beta 1$ protein expression and promotes gastric cancer cell invasiveness through the formyl peptide receptor/ERK/integrin $\beta 1$ pathway. Integrins also recruit and regulate paxillin expression in multiple cells [12,13]. ANXA1 and ANXA2 share similar functions, given their structural similarities. Thus, ANXA2 and paxillin could regulate the expression of cytoskeletal proteins, which results in the migration and adhesion of PASMCs $[14,15,16]$.

The purpose of this work was to examine the role of ANXA2 in regulating the migration of PASMCs following exposure to HPS rat serum and to identify the underlying mechanism[17,18]. We established the HPS model, detected the expression of ANXA2 in PASMCs, and found increased migration of PASMCs after treatment with HPS rat serum. We also detected an interaction between ANXA2 and paxillin. Our data showed that the expression of ANXA2 in PASMCs increased significantly, while the migration of PASMCs was noticeably enhanced when the cells were stimulated with HPS rat serum. In addition, ANXA2 and paxillin co-immunoprecipitated. Upon knockdown of ANXA2 expression, we observed decreased paxillin expression and a reduction in PASMC migration.

\section{Materials and Methods}

\subsection{HPS rat model and cell culture}

All procedures performed on the rats were conducted according to guidelines from the National Institutes of Health. The study protocol was approved by the committee on Animal Research of Southwest Hospital. Sprague Dawley (SD) rats weighing 180-220 g, which were obtained from the Laboratory Animal Center of the Third Military Medical University, were used in this study. The HPS rat model was used in our previously published study successfully [19]. The protocol utilised here was similar to the previous studies. The HPS rat model is defined based on a combination of gas exchange abnormalities $\left(\mathrm{PaO}_{2}<85 \mathrm{mmHg}\right.$ and $\left.\mathrm{P}(\mathrm{A}-\mathrm{a}) \mathrm{O}_{2}>18 \mathrm{mmHg}\right)$ and intrapulmonary vascular dilation found in pathological sections. HPS rat serum was collected from rats that had developed HPS. Normal rat serum was collected from the control rats at the same time. All of the serum samples were used for subsequent experiments. PASMCs were isolated from SD rat lungs as previously described [20]. PASMCs were cultured at a concentration of $106 / \mathrm{cm}^{2}$ and were divided into two groups: group C consisted of PASMCs that were cultured in 
Dulbecco's modified Eagle's medium (DMEM, Invitrogen, Carlsbad, CA, USA) supplemented with normal rat serum (5\%) and group HPS consisted of PASMCs that were incubated in DMEM containing 5\% HPS rat serum for $36 \mathrm{~h}$ (T1), $48 \mathrm{~h}$ (T2) and $72 \mathrm{~h}$ (T3).

\subsection{Scratch wound motility assay}

The rat PASMCs were seeded in 6-well plates $(1.50 \times 105$ cells/well) and grown to confluence. Twenty-four hours after serum deprivation, the cells from each group were mounted on a reusable template to create a standard wound $(<5 \mathrm{~mm})$. The migration status of PASMCs transfected by siANXA2 was observed by a fluorescence inverted microscope. Then, the reticule in the microscope was used to measure the planar migration of the cells in each group at 36,48 and $72 \mathrm{~h}$ after the scratch was made.

\subsection{Modified Boyden chamber}

The rat PASMCs were seeded in 24-well Transwell chambers with fibronectin-coated polycarbonate membranes (BD Bioscience, USA). The lower wells of the chamber were filled with DMEM and 20\% foetal calf serum (FCS) and the upper wells did not contain FCS. PASMCs were allowed to migrate for $24 \mathrm{~h}$ at $37^{\circ} \mathrm{C}$. The cells that were associated with the upper surface of the filters were gently removed, and the cells that were associated with the lower surface of the filters were fixed with paraformaldehyde. Chemotaxis was quantified by counting cells on the lower surface of the filter in one representative high-power field in each well using a grid that contained 100 non-overlapping fields. Experiments were performed at least four times.

\subsection{Reverse transcription PCR (RT-PCR) analysis}

Total RNA was extracted using the Tripure isolation reagent according to the manufacturer's instructions. Reverse transcription was performed with $2 \mu \mathrm{g}$ of RNA, Oligo (dT) (CWBIO, Beijing, China), dNTPs (CWBIO, Beijing, China), a primer mix and the reaction buffer supplied with the MMLV reverse transcriptase (CWBIO, Beijing, China). The fluorescence intensity ratio of the target gene compared with that of GAPDH was used as a measure of the relative gene expression. The polymerase chain reaction (PCR) amplification reaction conditions were as follows: $94^{\circ} \mathrm{C}$ for $2 \mathrm{~min}$, followed by 32 cycles of denaturation at $94^{\circ} \mathrm{C}$ for $30 \mathrm{~s}$, annealing at 
$62.5^{\circ} \mathrm{C}$ for $30 \mathrm{~s}$, elongation at $72^{\circ} \mathrm{C}$ for $30 \mathrm{~s}$, and a final termination step at $72^{\circ} \mathrm{C}$ for $10 \mathrm{~min}$. The relative quantification of ANXA2 mRNA levels was normalised to that of the internal control gene GAPDH. The experiment was performed independently four times, and the relative amount of each target gene was quantified. Primer sequences for ANXA2 and GAPDH are indicated in Table 1.

\subsection{Western blot}

Standard techniques were used to collect cells. The cultured cells were lysed, and total protein was extracted according to the manufacturer's instructions. The BCA protein assay (CWBIO, Beijing, China) was used to quantify the amount of protein. Equal quantities of proteins were separated with sodium dodecyl sulphate-polyacrylamide gel electrophoresis (SDS-PAGE, CWBIO, Beijing, China) and transferred to PVDF membranes. The membranes were blocked for $1 \mathrm{~h}$ using a blocking solution. The samples were incubated overnight with the following primary antibodies: mouse monoclonal anti-ANXA2 (RD Systems, Minneapolis, USA) and rabbit polyclonal anti-paxillin (Abcam, Cambridge, MA, UK), at a dilution of 1:1000 at $4^{\circ} \mathrm{C}$. The membrane was washed twice with TBS, and the bound antibody was detected using the following IgG-HRP secondary antibodies: goat anti-rabbit $\operatorname{IgG}$ and goat anti-mouse $\operatorname{IgG}$ (Santa Cruz, CA, USA), at a dilution of $1: 1000$ for $1 \mathrm{~h}$ at room temperature. Finally, the membranes were stained with diaminobenzidine, scanned, and stored using a gel imaging system. The optical density of each immunoreactive band was measured and analysed with an Alpha Imager.

\subsection{Transfection of ANXA2 siRNA into PASMCs}

The transfection technique was similar to the technique used in previous studies. The ANXA2-specific siRNA and siRNA negative control were purchased from SBO Medical Biotechnology (Shanghai, China), and the target sequences are shown in Table 2. Both of the siRNAs were transfected with EntransterTM-R (Engreen Biosystem, Beijing, China). Approximately $10 \times 10^{5} \pm 2.5 \times 10^{5}$ PASMCs were seeded into a $100 \mathrm{~mm}$ culture dish in $10 \mathrm{ml}$ of complete medium $24 \mathrm{~h}$ prior to transfection. siRNA was transfected into the PASMCs using the EntransterTM-R transfection reagent with a final concentration of $100 \mathrm{nM}$. Twenty-four hours after transfection, the PASMCs were incubated with either normal rat serum or HPS rat serum for 
various time periods $(36,48$, and $72 \mathrm{~h})$. The efficacy of ANXA2 silencing on the different groups of cells was assessed by RT-PCR and western blotting after $60 \mathrm{~h}$ of transfection.

\subsection{Co-immunoprecipitation}

The rat PASMCs were lysed for $1 \mathrm{~h}$ at $4^{\circ} \mathrm{C}$ using RIPA lysis buffer (Beyotime, Shanghai, China). Cell debris was pelleted at $14,000 \mathrm{~g}$ for $5 \mathrm{~min}$. Some of the supernatant was incubated with a rabbit anti-paxillin polyclonal antibody; these samples were in the group paxillin(+). The other set of supernatants was incubated with PBS, and were called group paxillin(-). All of the samples were incubated overnight at $4^{\circ} \mathrm{C}$. Then, $40 \mu \mathrm{L}$ of protein $\mathrm{A}+\mathrm{G}$ agarose (Beyotime, Shanghai, China) was added to the samples for $3 \mathrm{~h}$ at $4^{\circ} \mathrm{C}$. After washing, $40 \mu \mathrm{L}$ SDS-PAGE was added into samples. Transfer onto PVDF membrane and immunoblotting were performed using standard procedures. At the same time, total proteins were extracted from PASMCs by conventional process of extracting proteins, and these were also run in a gel and transferred onto PVDF membrane to serve as a control (group C). Finally, we captured an image of the PVDF membrane with an Alpha Imager. The mouse anti-ANXA2 monoclonal antibody was used at a dilution of 1:500 and the HRP-conjugated goat anti-mouse IgG was diluted 1:1000. The blots were developed with the ECL reagent (Beyotime, Shanghai, China).

\subsection{Statistical Analysis}

Results are shown as the mean \pm SEM. One-way ANOVA and paired t-tests were performed for statistical analysis as appropriate. A p-value $<0.05$ was considered statistically significant.

\section{Results}

\subsection{HPS rat serum enhanced the migration of PASMCs, as identified by the scratch} wound motility assay and the modified Boyden chamber

The results of scratch wound motility assay indicated that treatment with normal rat serum did not increase the distance that PASMCs migrated, which remained consistently low at each time-point. However, the distance PASMCs migrated increased significantly when the PASMCs had been stimulated with HPS rat serum. The distance increased in a time-dependent manner (Fig. 1A). The modified Boyden chamber results showed that HPS rat serum promoted transmembrane 
migration of PASMCs compared with treatment with normal rat serum (Fig. 1B).

3.2 HPS rat serum enhanced the mRNA and protein expression levels of ANXA2 in the PASMCS

The ANXA2 mRNA and protein levels were detected in the PASMCs from all groups. ANXA2 transcription was detected at low level in the PASMCs treated with normal rat serum. However, treatment with HPS rat serum increased the ANXA2 mRNA level significantly, and the increase correlated with the treatment time. At the same time-points, ANXA2 mRNA levels in group HPS were significantly higher than in group C (Fig. 1C). The western blotting results showed that treatment with normal rat serum did not increase ANXA2 expression, but after exposure to HPS rat serum, the expression of ANXA2 increased in a time-dependent manner (Fig. 1D). The GAPDH expression level was similar in all groups.

\section{3 siRNA effectively downregulated ANXA2 expression}

The RT-PCR results revealed that there was a prominent reduction in the ANXA2 expression in siANXA2-treated cells compared with cells in the siControl of group C and group HPS $60 \mathrm{~h}$ after siRNA transfection. The results showed that the mRNA level of ANXA2 was decreased by $>90 \%$ after using the ANXA2 siRNA (Fig. 2A). Western blotting showed that more than $60 \mathrm{~h}$ after siANXA2 transfection, the expression of ANXA2 decreased in siANXA2-treated cells compared with cells treated with the siControl from both groups. More than $90 \%$ of the ANXA2 protein expression was effectively inhibited after transfection of the ANXA2 siRNA into cells from both group C and group HPS (Fig. 2B).

\subsection{ANXA2 and paxillin co-immunoprecipitate}

The ANXA2 protein could be detected in the complex of group paxillin(+), which included the rabbit anti-paxillin antibody, and in lysates from group C. The expression of ANXA2 could not be detected in the complex of paxillin(-), which did not include the rabbit anti-paxillin antibody. We confirmed that the immunoprecipitating protein was in fact ANXA2 by its molecular weight and by comparing the band to that seen in samples from group C. The results showed that ANXA2 and paxillin interact with one another and co-precipitate (Fig. 3A). 


\subsection{Silencing ANXA2 expression suppressed the HPS rat serum-induced up-regulation}

in paxillin expression

After the transfection of siANXA2, the HPS rat serum-induced increase in paxillin expression was clearly inhibited. There was a remarkable increase in the paxillin expression in group HPS compared with group $\mathrm{C}$ at the same time-point in cells that had been transfected with siControl. The expression of paxillin increased in a time-dependent manner after treatment with the HPS serum. However, after transfection with siANXA2 following HPS rat serum exposure, the paxillin expression did not increase, and there was no significant difference in paxillin expression in siANXA2-group HPS and ANXA2-group C. The paxillin expression was at low in both groups (Fig. 3B).

3.6 Silencing ANXA2 expression suppressed the HPS rat serum-induced migration of PASMCS

The increase in the distance cells migrated induced by HPS rat serum was inhibited when PASMCs were transfected with siANXA2. When the cells were transfected with siControl, the distance of cell migration in group HPS was higher than that in group $\mathrm{C}$ at each time-point. HPS rat serum increased the distance of cell migration significantly. After exposure of the PASMCs to HPS rat serum, there was a significant reduction in the distance cells migrated in group siANXA2 compared with group siControl (Fig. 4A). We counted the number of cells that migrated through the modified Boyden chamber. The results showed that HPS rat serum promoted transmembrane migration, and the transduction of siANXA2 significantly inhibited the HPS rat serum-induced PASMCs transmembrane migration (Fig. 4B).

\section{Discussion}

Previous studies and our initial study demonstrated that HPS rat serum induces PASMC proliferation and that hypoxia enhances the migration of PASMCs[21,22]. These are key pathophysiological components of PVR associated with HPS [23]. It 
has been reported that the migration of vascular smooth muscle cells (VSMCs) in some organs or tissues is a key early change in vascular remodelling $[8,9]$.

Our data indicated that HPS rat serum enhanced PASMC migration dramatically. Recent research has shown that the migration of PASMCs is critical for physiological and pathological changes in multiple blood vessels that occur during HPS. Furthermore, it was demonstrated that PASMC migration occurs before their proliferation when the cells are stimulated with hypoxia or by numerous circulating cytokines $[8,15,20]$ Our findings suggested that HPS rat serum up-regulated PASMC migration levels. The activation of PASMC migration subsequently leads to an enhanced the proliferation of these cells, which can give rise to the development of PVR in HPS. However, the molecular mechanisms have not been defined. It would be useful to define the key molecular target responsible for HPS-associated increased PASMC migration[24,25,26].

Our findings showed that ANXA2 is expressed at higher levels in PASMCs treated with HPS rat serum than in those treated with normal rat serum. PASMC migration is also enhanced by HPS rat serum. This increase in ANXA2 was observed on both the transcription and protein levels. Due to existing evidence on the changes in ANXA2 expression and the migration of PASMCs in HPS-associated PVR, we sought to investigate whether specifically reducing the levels of ANXA2 could result in inhibition of PASMC migration. It was possible that ANXA2 was acting as a key regulator in the migration of PASMCs in HPS-associated PVR.

Our data indicated that ANXA2 and paxillin directly interacted with one another. Furthermore, silencing ANXA2 expression by transfection with ANXA2 siRNA suppressed the HPS rat serum-induced up-regulation of paxillin expression in PASMCs[7]. Furthermore, we found that silencing paxillin expression could reverse the migration of PASMCs induced by HPS rat serum. This finding suggests that ANXA2 acts a regulator in paxillin expression and PASMC migration in HPS[27]. 
In conclusion, our data showed that the expression of ANXA2 and the migration of PASMCs increased dramatically when the cells were treated with HPS rat serum. Furthermore, we found that inhibiting ANXA2 expression by siRNA suppressed the expression of paxillin and the migration of HPS rat serum-treated PASMCs. All together, these results demonstrate that silencing ANXA2 expression provides a potential mechanism of protection. These findings indicated that ANXA2 plays a key regulatory role in the pathogenesis of PVR in HPS. In the future, it will be useful to understand the mechanisms of abnormal cell migration to provide a basis for inhibiting this process and creating targeted therapies for diseases associated with PVR.

\section{Acknowledgments}

This work is supported by grant nos. $30700347,30872448,81170053,81170414$ and 81270510 from the National Science Foundation of China (NSFC).

\section{References}

[1] J. Zhang, W. Yang, B. Luo, B. Hu, A. Maheshwari, M.B. Fallon, The role of CX(3)CL1/CX(3)CR1 in pulmonary angiogenesis and intravascular monocyte accumulation in rat experimental hepatopulmonary syndrome, J Hepatol 57 (2012) 752-758.

[2] A. Miyamoto, Y. Katsuta, X.J. Zhang, H.L. Li, M. Ohsuga, H. Komeichi, S. Shimizu, T. Akimoto, K. Mizuno, Effect of chronic methylene blue administration on hypoxemia in rats with common bile duct ligation, Hepatol Res 40 (2010) 622-632.

[3] J. Zeng, B. Yi, Z. Wang, B. Guo, K. Lu, X. Wang, Role of annexin A1 in human pulmonary arterial smooth muscle cells proliferation induced by hypoxia, Chin J Anesthesiol 31 (2011) 1239-1241.

[4] G.S. Wang, G.S. Qian, D.S. Zhou, J.Q. Zhao, JAK-STAT signaling pathway in pulmonary arterial smooth muscle cells is activated by hypoxia, Cell Biol Int 29 (2005) 598-603.

[5] C. Luo, B. Yi, L. Bai, Y. Xia, G. Wang, G. Qian, H. Feng, Suppression of Akt1 phosphorylation by adenoviral transfer of the PTEN gene inhibits hypoxia-induced proliferation of rat pulmonary arterial smooth muscle cells, Biochem Biophys Res Commun 397 (2010) 486-492.

[6] C. Veith, L.M. Marsh, M. Wygrecka, K. Rutschmann, W. Seeger, N. Weissmann, G. Kwapiszewska, Paxillin regulates pulmonary arterial smooth muscle cell function in pulmonary hypertension, Am J Pathol 181 (2012) 1621-1633.

[7] B. Yi, J. Cui, J. Ning, J. Gu, G. Wang, L. Bai, G. Qian, K. Lu, cGMP-dependent protein kinase lalpha transfection inhibits hypoxia-induced migration, phenotype modulation and annexins A1 expression in human pulmonary artery smooth muscle cells, Biochem Biophys Res Commun 
418 (2012) 598-602.

[8] Y. Chen, B. YI, B. Chen, Y. Yang, B. Zu, K. Lu, Changes in expression of annexin A2 in lung tissues in rats with hepato-pulmonary syndrome Chin J Anesthesiol 33 (2013) 599-601.

[9] J. Zeng, B. Yi, Z. Wang, J. Ning, X. Wang, K. Lu, Effect of annexin A2 on hepatopulmonary syndrome rat serum-induced proliferation of pulmonary arterial smooth muscle cells, Respir Physiol Neurobiol 185 (2013) 332-338.

[10] P.A. Madureira, A.P. Surette, K.D. Phipps, M.A. Taboski, V.A. Miller, D.M. Waisman, The role of the annexin A2 heterotetramer in vascular fibrinolysis, Blood 118 (2011) 4789-4797.

[11] M.C. Derry, M.R. Sutherland, C.M. Restall, D.M. Waisman, E.L. Pryzdial, Annexin 2-mediated enhancement of cytomegalovirus infection opposes inhibition by annexin 1 or annexin 5, J Gen Virol 88 (2007) 19-27.

[12] T.Y. Cheng, M.S. Wu, J.T. Lin, M.T. Lin, C.T. Shun, H.Y. Huang, K.T. Hua, M.L. Kuo, Annexin A1 is associated with gastric cancer survival and promotes gastric cancer cell invasiveness through the formyl peptide receptor/extracellular signal-regulated kinase/integrin beta-1-binding protein 1 pathway, Cancer 118 (2012) 5757-5767.

[13] N.O. Deakin, J. Pignatelli, C.E. Turner, Diverse roles for the paxillin family of proteins in cancer, Genes Cancer 3 (2012) 362-370.

[14] L. Tcatchoff, S. Andersson, A. Utskarpen, T.I. Klokk, S.S. Skanland, S. Pust, V. Gerke, K. Sandvig, Annexin A1 and A2: roles in retrograde trafficking of Shiga toxin, PLoS One 7 (2012) e40429.

[15] Y. Chen, B. Yi, Z. Wang, J. Gu, Y. Li, J. Cui, K. Lu, Paxillin suppresses the proliferation of HPS rat serum treated PASMCs by up-regulating the expression of cytoskeletal proteins, Molecular BioSystems 10 (2014) 756-766.

[16] B. Yi, J. Zeng, G. Wang, G. Qian, K. Lu, Annexin A1 protein regulates the expression of PMVEC cytoskeletal proteins in CBDL rat serum-induced pulmonary microvascular remodeling, J Transl Med 11 (2013) 98-106.

[17] B. Chen, J.L. Ning, J.T. Gu, J. Cui, Y. Yang, Z. Wang, J. Zeng, B. Yi, K.Z. Lu, Caspase-3 inhibition prevents the development of hepatopulmonary syndrome in common bile duct ligation rats by alleviating pulmonary injury, Liver Int 35 (2015) 1373-1382.

[18] T. Thenappan, A. Goel, G. Marsboom, Y.H. Fang, P.T. Toth, H.J. Zhang, H. Kajimoto, Z. Hong, J. Paul, C. Wietholt, J. Pogoriler, L. Piao, J. Rehman, S.L. Archer, A central role for CD68(+) macrophages in hepatopulmonary syndrome. Reversal by macrophage depletion, Am J Respir Crit Care Med 183 (2011) 1080-1091.

[19] B. Guo, B. Yi, S. Xu, K. Lu, Effect of serum obtaied from rat with hepatopulmomry syndrome on Akt mRNA and protein expression in rat pulmonary mlcrovascular endothelial cells, Chin J Anesthesiol 30 (2010) 75-78.

[20] B. Yi, J. Cui, J.N. Ning, G.S. Wang, G.S. Qian, K.Z. Lu, Over-expression of PKGlalpha inhibits hypoxia-induced proliferation, Akt activation, and phenotype modulation of human PASMCs: the role of phenotype modulation of PASMCs in pulmonary vascular remodeling, Gene 492 (2012) 354-360.

[21] L. Liu, N. Liu, Z. Zhao, J. Liu, Y. Feng, H. Jiang, D. Han, TNF-alpha neutralization improves experimental hepatopulmonary syndrome in rats, Liver Int 32 (2012) 1018-1026.

[22] S. Gupta, M.E. Faughnan, L. Lilly, S. Hutchison, R. Fowler, A.M. Bayoumi, Norfloxacin therapy for hepatopulmonary syndrome: a pilot randomized controlled trial, Clin Gastroenterol Hepatol 8 (2010) 1095-1098. 
[23] Z. Wang, B. Yi, B. Guo, K. Lu, Changes in expression of transforming growth factor beta-1 during myogenic differentiation of pulmonary microvascular endothelial cells in rats with hepato-pulmonary syndrome, Chin J Anesthesiol 31 (2011) 862-864.

[24] T. Grewal, C. Enrich, Annexins--modulators of EGF receptor signalling and trafficking, Cell Signal 21 (2009) 847-858.

[25] Y. Huang, Y. Jin, C.H. Yan, Y. Yu, J. Bai, F. Chen, Y.Z. Zhao, S.B. Fu, Involvement of Annexin A2 in p53 induced apoptosis in lung cancer, Mol Cell Biochem 309 (2008) 117-123.

[26] H. Bao, M. Jiang, M. Zhu, F. Sheng, J. Ruan, C. Ruan, Overexpression of Annexin II affects the proliferation, apoptosis, invasion and production of proangiogenic factors in multiple myeloma, Int J Hematol 90 (2009) 177-185.

[27] M.C. Brown, C.E. Turner, Paxillin: adapting to change, Physiol Rev 84 (2004) 1315-1339. 


\section{Fig. 1.}

Effect of normal rat serum or HPS rat serum on PASMC migration. (A) The distance PASMCs migrated in a dish following treatment with normal rat serum or HPS rat serum (mm; *p < 0.05 vs. Group C or Group HPS T1; ${ }^{*} \mathrm{p}<0.05$ vs. Group HPS T2). (B) HPS rat serum enhanced PASMC transmembrane migration (*p<0.05 vs. Group C). (C,D) Levels of ANXA2 mRNA and protein in PASMCs treated with normal rat serum or HPS rat serum. Exposure to HPS rat serum significantly increased the expression of ANXA2 mRNA and protein levels in the PASMCs (\%; ${ }^{*} \mathrm{p}<0.05$ vs. Group C; ${ }^{*} \mathrm{p}<0.05$ vs. Group HPS T1; ${ }^{\circledR} \mathrm{p}<0.05$ vs. Group HPS T2). Each data point represents the mean \pm S.E.M. of four independent experiments.

\section{Fig. 2.}

Levels of ANXA2 mRNA and protein in PASMCs that were incubated with normal serum or HPS rat serum and treated concurrently with ANXA2 siRNA. (A) The ANXA2 mRNA levels were dramatically diminished after $60 \mathrm{~h}$ of transfection compared with levels in the Group siControl. (B) After $60 \mathrm{~h}$ of transfection, the protein expression of ANXA2 was strongly suppressed by ANXA2 siRNA (\%; ${ }^{*}$ p $<0.05$ vs. Group siControl). Each data point represents the mean \pm S.E.M. of four independent experiments.

\section{Fig. 3.}

ANXA2 and paxillin co-precipitate, as determined by a co-immunoprecipitation assay. (A) Images of the immunoblot for the ANXA2 and paxillin co-immunoprecipitation. Levels of paxillin mRNA and protein in PASMCs which were treated with normal serum or HPS rat serum and with siANXA2. (B)The HPS rat serum-induced up-regulation of paxillin in PASMCs was inhibited by transfection with siANXA2 (\%; *p $<0.05$ vs. siControl in Group $\mathrm{C}$ or siANXA2; \#P< 0.05 vs. siControl in Group HPS T1; §P $<0.05$ vs. siControl in Group HPS T2). Each data point represents the mean \pm S.E.M. of four independent experiments. Group $\mathrm{C}$ in part A: Total proteins in PASMCs were extracted by a conventional process; Group- $\mathrm{P}(+)$ : lysed proteins incubated with rabbit anti-paxillin polyclonal antibody; Group-P(-): lysed proteins incubated with PBS only.

\section{Fig. 4.}

Effect of siANXA2 transfection on PASMC migration after the cells were treated with normal rat serum or HPS rat serum. (A) The distance PASMCs migrated on a flat surface following treatment with normal rat serum or HPS rat serum and treated concurrently with siANXA2 (mm; *p<0.05 vs. siControl in Group C; \#p<0.05 vs. siANXA2 in Group HPS). (B) siANXA2 suppressed the PASMC transmembrane migration induced by HPS rat serum (*p $<0.05$ vs. Group $\mathrm{C}$ or siANXA2 in Group HPS). Each data point represents the mean \pm S.E.M. of four independent experiments. SiANXA2-C: Group C with siANXA2; siANXA2-H: Group HPS with siANXA2; siControl-C: Group C with siControl; siControl-H: Group HPS with siControl. 
Table 1

The primer sequences of ANXA2 and GAPDH.

\begin{tabular}{llll}
\hline Gene name & Primer sequences & Bp & Annealing temperature \\
\hline ANXA2 & F:5'-TGCCTATGGGTCGGTCAAAC-3' & 154 & $62.5^{\circ} \mathrm{C}$ \\
& R:5'-GAAGGCAATGTCCTGCCTCT-3' & & \\
\multirow{2}{*}{ GAPDH } & F:5'-TACGACAGTCCATGCCATCAC-3' & 493 & $56^{\circ} \mathrm{C}$ \\
& R:5'-TCCACCACCTGTGGCTGTA-3' & & \\
\hline
\end{tabular}


Table 2

The target sequences of ANXA2 siRNA and negative siRNA.

\begin{tabular}{ll}
\hline Gene name & Target sequence \\
\hline ANXA2 siRNA & GGAAATGTACAAGACCGAT \\
Negative control siRNA & GGAGTATACGACACAAGAT \\
\hline
\end{tabular}


A $\square$ Group $\mathrm{m}$ Group HPS

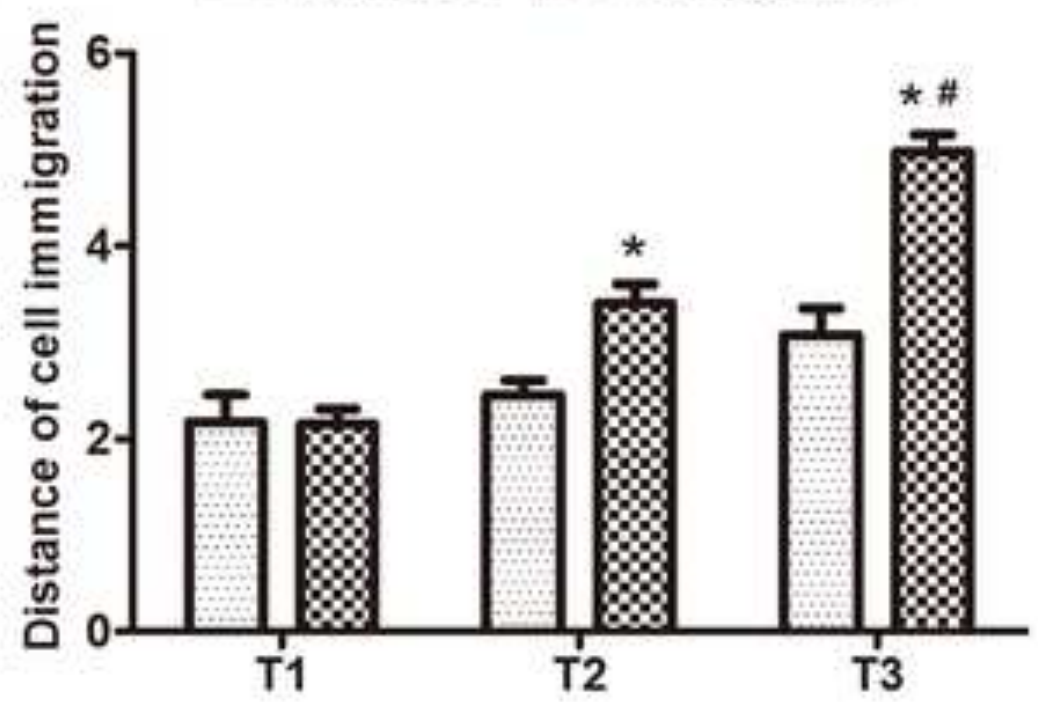

C

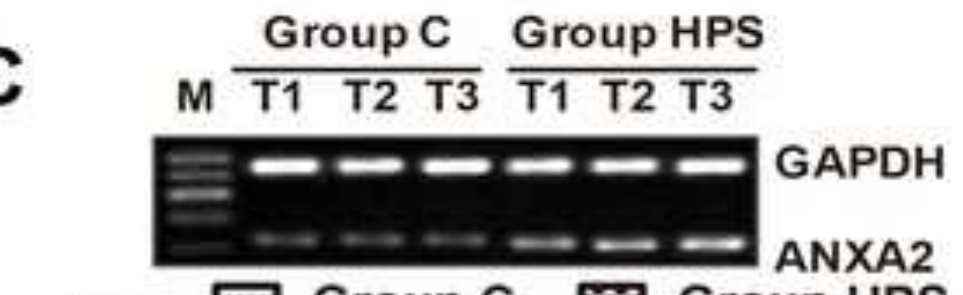

B $\square$ Group C $\bowtie$ Group HPS

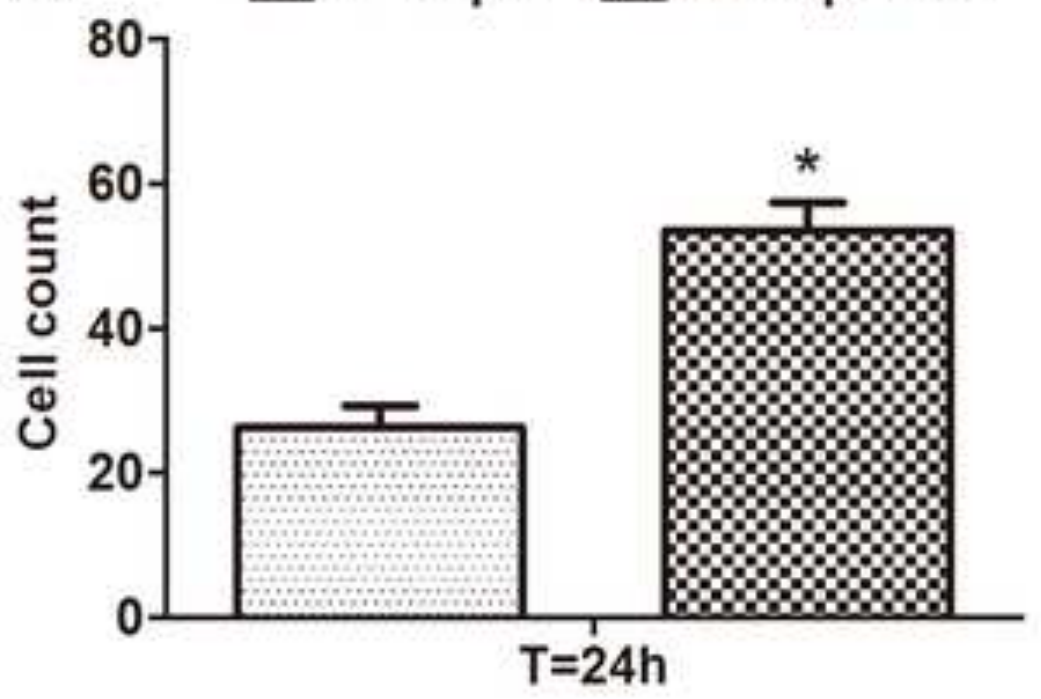

D $\quad \frac{\text { Group C }}{\mathrm{T} 1 \mathrm{~T} 2 \mathrm{~T} 3} \frac{\text { Group HPS }}{\mathrm{T} 1 \mathrm{~T} 2 \mathrm{~T} 3}$

ANXA2

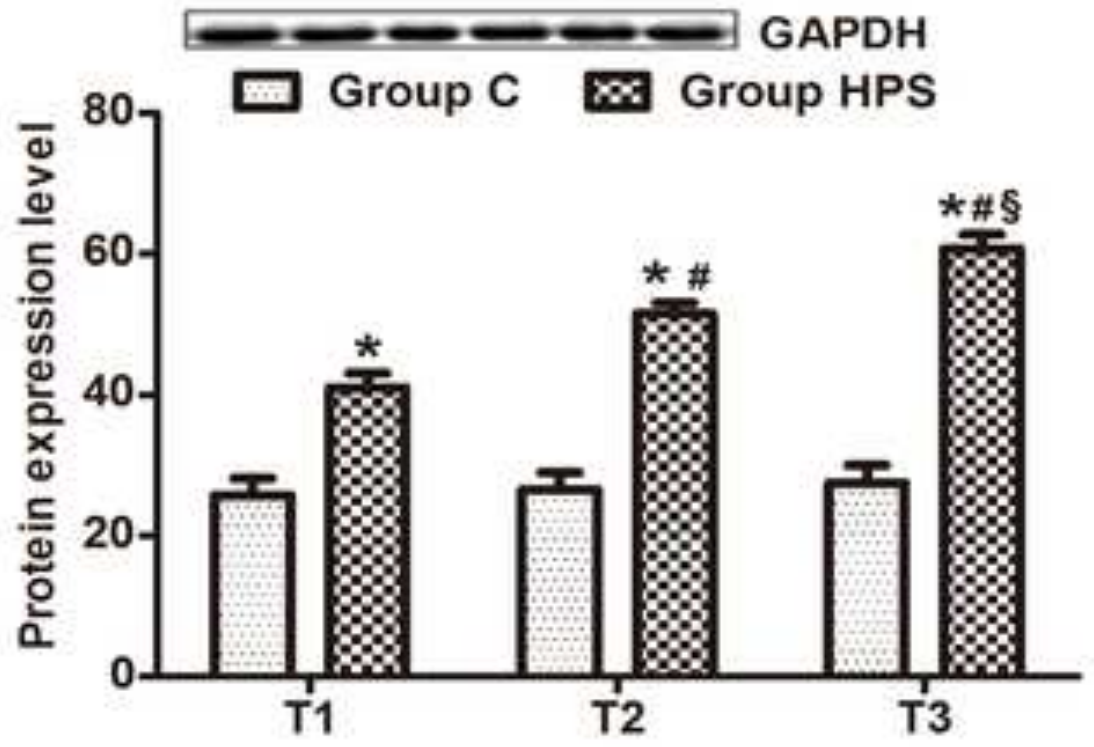


A

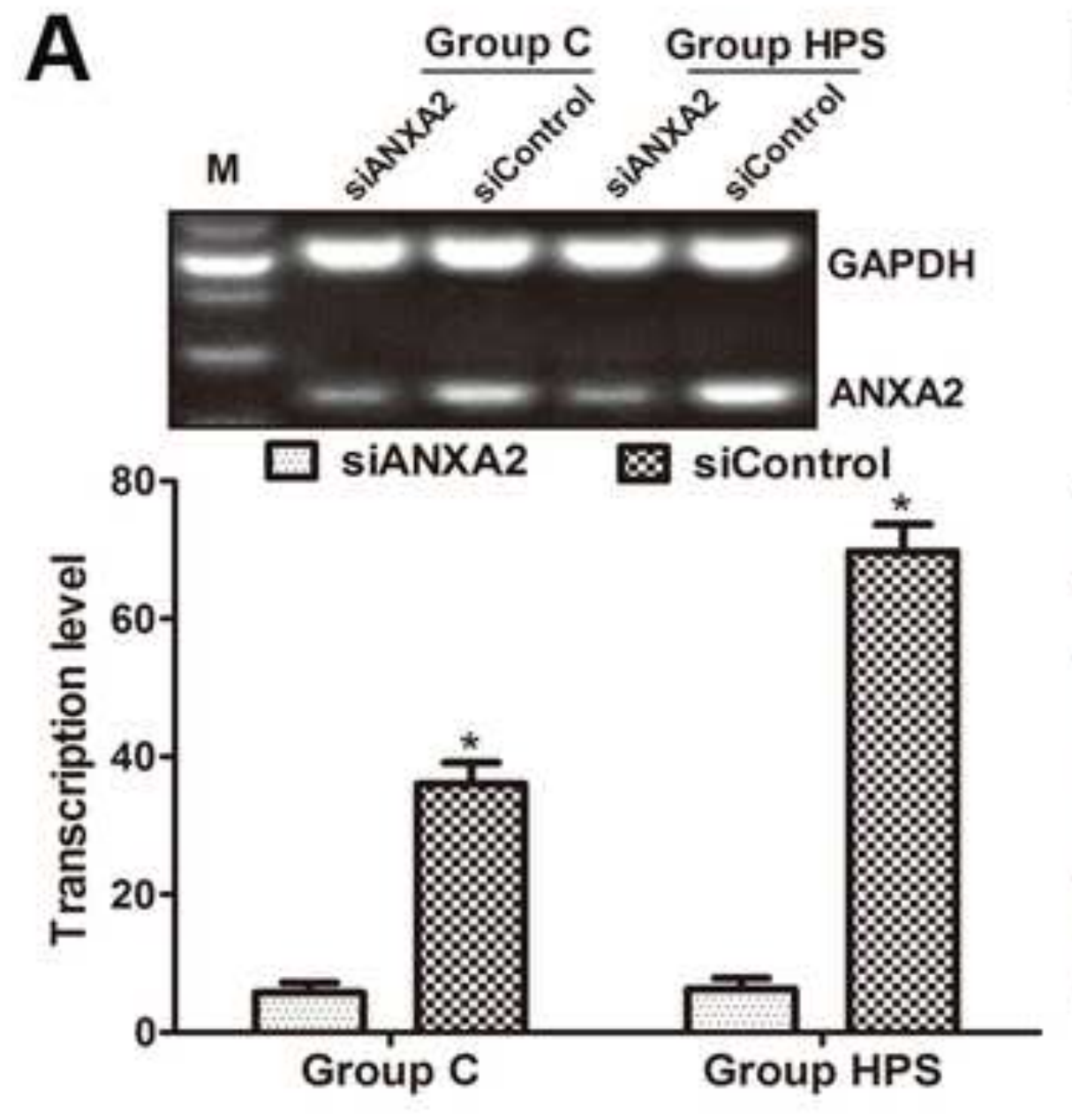

B
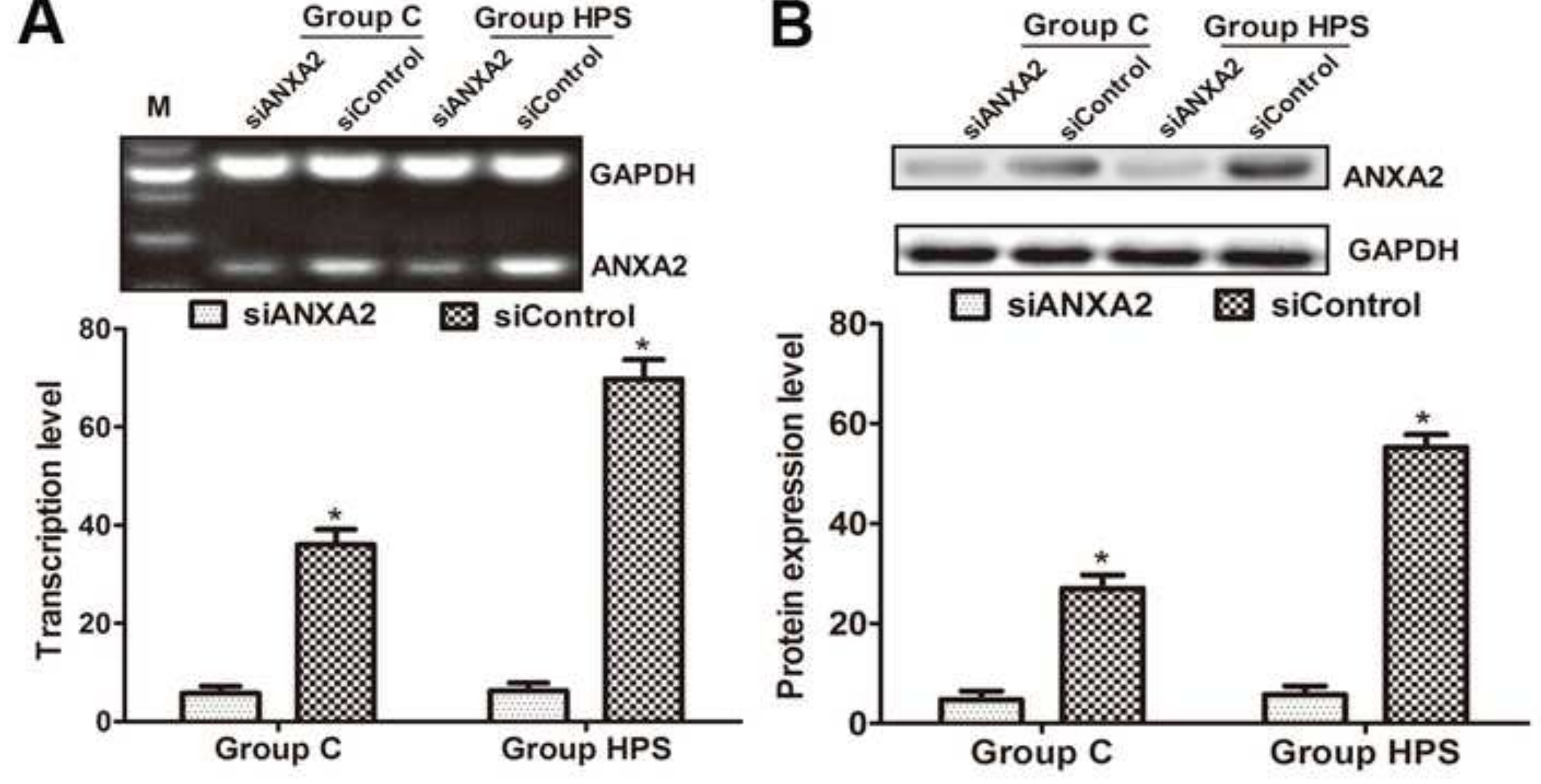

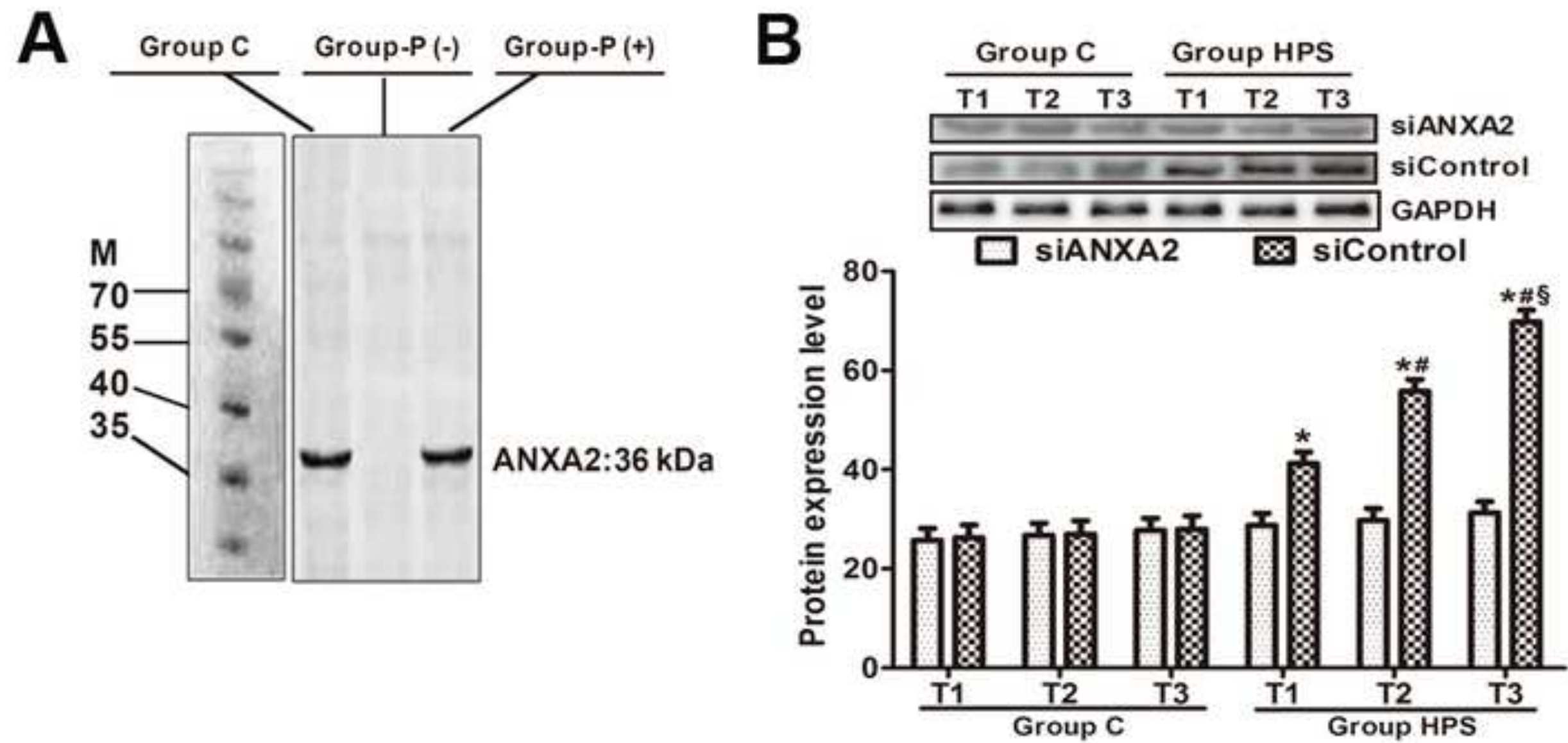


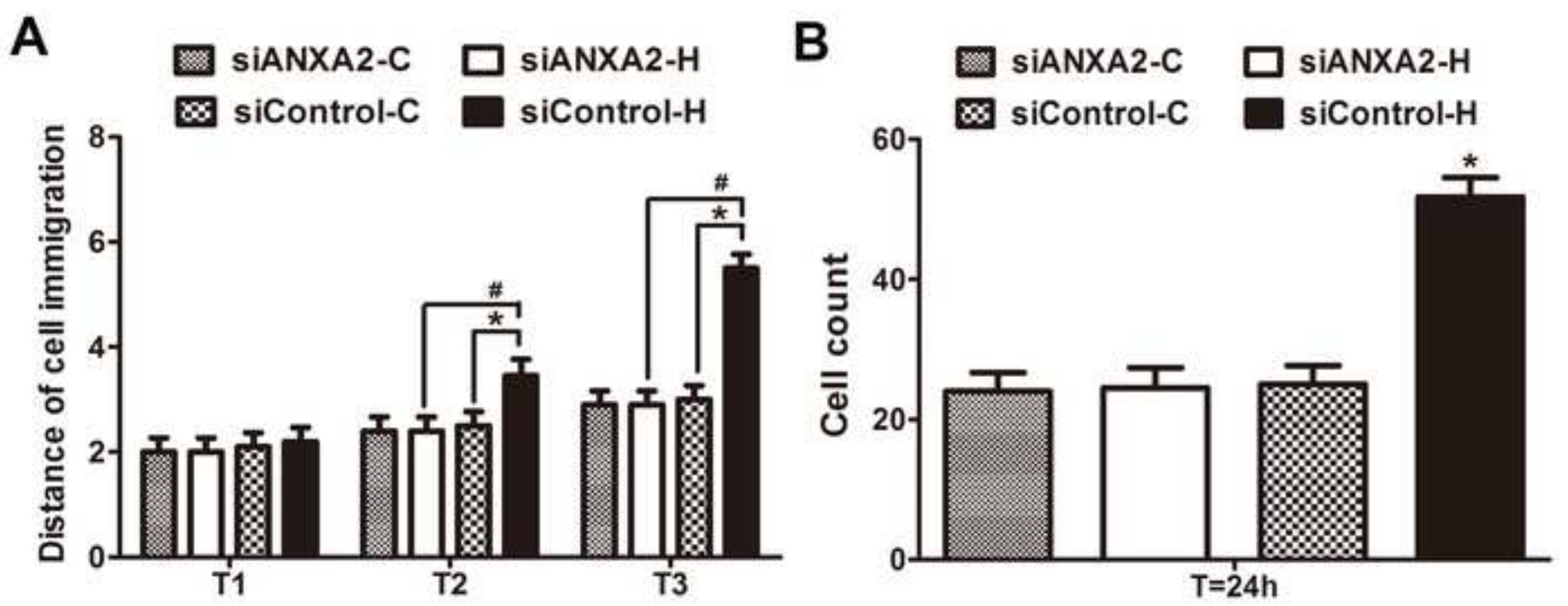

